\title{
ANN-based Classifier for PAF Prediction
}

\author{
Ashraf Anwar Fahmy \\ College of Computers and Information Technology, \\ Taif University, Saudi Arabia
}

\author{
Fahad Al Raddady \\ College of Computers and Information Technology, \\ Taif University, Saudi Arabia
}

\begin{abstract}
In this paper we propose an artificial neural network as a based classifier for prediction of Paroxysmal Atrial Fibrillation (PAF). PAF is a really life threatening disease and it is the result of irregular and repeated depolarization of the atria. We used PAF prediction data base which include 30 -min. period of 100 ECG recorded signals. We divide the 30 -min preceding the PAF into 6 periods with 5-min each. In each suggested period we get the classification result using ANN. The results show that we can predict the PAF accurately in 5-min \& 20-min prior the PAF. In these two periods, the measured sensitivity, specificity, positive predictivity and accuracy show better and significant results comparable to the other periods. Also the results outperform the obtained results in the same field in the literature.
\end{abstract}

\section{Keywords}

PAF prediction, ECG signal, continuous wavelet transform, artificial neural network (ANN), Feature Extraction.

\section{INRODUCTION}

Classification of ECG signal is an important area in biomedical signal processing. Paroxysmal atrial fibrillation (PAF) of the heart muscle is defined as short duration episodes of AF lasting from $2 \mathrm{~min}$. to less than 7 days, while chronic $\mathrm{AF}$ is defined as lasting more than 7 days. The main reason for this is not the immediate effect of the onset of atrial fibrillation over the patient's health (AF detection) but the long-term effects: increase in heart muscle fatigue, increase in thromboembolic and stroke events due to the formation of blood clots and an irregular onset that makes it hard to detect on normal ECG tests. Thus it is necessary for cardiologists to benefit from a robust and precise tool that could predict the onset of such events, in order to prevent them by defibrillation, drug treatment and antitachycardia pacing techniques. Chronic AF is usually preceded by (PAF). Therefore, in addition to use anti-arrhythmic drugs, the physicians are trying to develop pacing devices in order to surpass the onset of AF.

The automated method to predict the onset PAF is interesting topic to help treating this problem. During recent years several researchers proposed many techniques to predict the onset of PAF. Useful reviews describing different techniques for PAF or chronic AF prediction, from technical to clinical points of view [1-4]. The "Computers in Cardiology Challenge 2001" revealed a maximum obtained accuracy of about $80 \%$ [5-7]. Hariton, et al., [8] proposed a new method for PAF automatic prediction based on heart rate variability (HRV) metrics and morphologic variability (MV), and (HRV+MV) decision rule, the obtained specificity and sensitivity are between (83.93\%- 89.29\%), (84.51\%-89,44\%) respectively. Artificial neural network (ANN) in recent years has proved to be an advanced tool in solving classification [9-11], wavelets proved usefulness in feature extraction from non-stationary signal like ECG [12-13]. In general, these above prediction models are able to detect the transition to PAF events with accuracies of $70-90 \%$, by means of records of at least tens of minutes and rather complex analysis procedures.
In the present work, two set of features are extracted: Feature set-1 (FS-1), directly from ECG signal and feature set-2 (FS-2) with the aid of continuous wavelet transform(CWT), which converts the time domain signal to time-frequency domain where several features can be carefully extracted, the extracted features are then applied to ANN to classify the normal object from that one who suffers from PAF.

\section{MATERIAL AND METHODS}

The database used for this task was PAF Prediction Challenge Database 2001 from physionet.org. It consists of 3 record sets: the first one has records that begin with the letter ' $n$ ' and comes from 50 subjects who do not have documented PAF. The length of these records is $30 \mathrm{~min}$. The second record that begin with letter ' $\mathrm{p}$ ' comes from 48 different subjects who have documented PAF, and it is divided into 50 record sets, the even one has a record of 30 min preceding the PAF, and the odd one has a record of 30 min. but distant from PAF. All the previous record has a continuation $5 \mathrm{~min}$ record with a letter ' $\mathrm{c}$ '. The third record contains 100 annotated recordings for testing with a letter't'

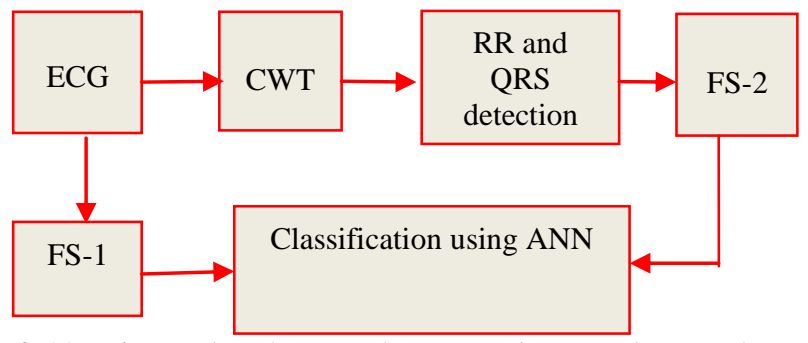

of $30 \mathrm{~min}$. and unknown documentation. Each record contains two channels simultaneous recorded Holter ECG signal digitized at $128 \mathrm{~Hz}$ with 12 bit resolution over a 20 $\mathrm{mV}$ range.

We used in this task both channels of the ECG signal, 50 normal channels and 50 PAF channel (25 even record $\mathrm{x} 2$ channel) to create the database.

The block diagram of the proposed method is shown in Fig. 1; each block is described in more details.

Fig. 1: Block diagram of the proposed method 


\subsection{Preprocessing.}

The ECG signal within the database can be affected by many interfering signals such as the $50 \mathrm{~Hz}$ power line interference and the baseline wandering. These interfering noises are eliminated first by means of a $5-15 \mathrm{~Hz}$ bandpass filter. For lowering interference noise, a median filter was used.

\subsection{Continuous Wavelet Transform (CWT)}

CWT allows a time domain signal to be transformed into time-frequency domain where frequency characteristics and the location of particular features in a time series may be highlighted simultaneously. Thus it allows accurate extraction of feature from non-stationary signal like ECG [14]. The CWT wavelet transform is a tool that divides up data, functions, or operators into different frequency components and then studies each component with a resolution matched to its scale. Unlike the short time Fourier transformation (STFT) the wavelet transformation has very good time and frequency resolution making it ideal in the analysis of non-stationary signals such as an ECG signal. The continuous wavelet transformation (CWT) of a signal $\mathrm{x}(\mathrm{t})$ is the convolution product of $\mathrm{x}(\mathrm{t})$ with a scaled and translated kernel function [15]

$$
\operatorname{CWT}_{\mathrm{x}}^{\emptyset}=\frac{1}{\sqrt{|\mathrm{s}|}} \int_{-\infty}^{\infty} \mathrm{x}(\mathrm{t}) \emptyset\left(\frac{\mathrm{t}-\tau}{\mathrm{s}}\right) \mathrm{dt}
$$

Where $\Phi((\mathrm{t}-\tau) / \mathrm{s})$ is a scaled and translated (shifted) version of a mother wavelet which is the basic unit of wavelet decomposition, $\mathrm{s}$ is a scale parameter and $\tau$ is a space parameter.

To analyze the CWT coefficients obtained for ECG signal of PAF record and non-PAF, predominant frequency vs. time plot of selected ECG signal has been obtained. From these plots translation, scale and coefficient values of the peaks, which represent $\mathrm{P}, \mathrm{Q}, \mathrm{R}, \mathrm{S}, \mathrm{T}$ and $\mathrm{U}$ wave has been extracted for PAF and non-PAF records. Fig. 2 and Fig. 4 show the normal ECG signal plot and the ECG signal preceding PAF plot respectively. Fig. 3 and Fig. 5 illustrate the difference in the amplitude and duration of RR and QRS among PAF and non-PAF records at different scales with the aid of CWT

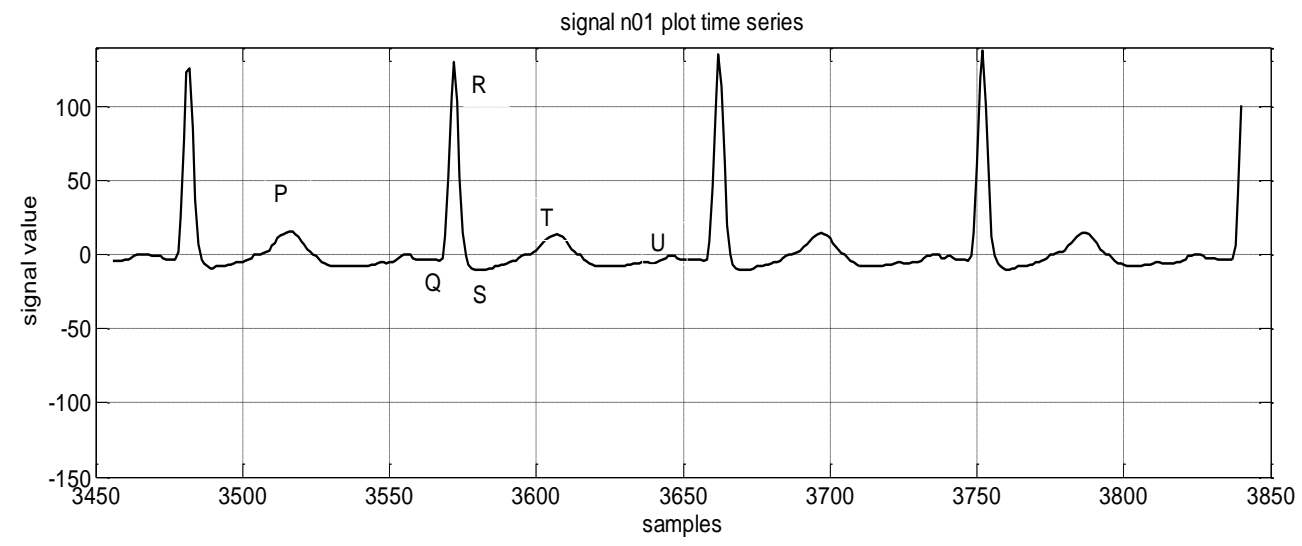

Fig. 2: Normal ECG signal (record n01)
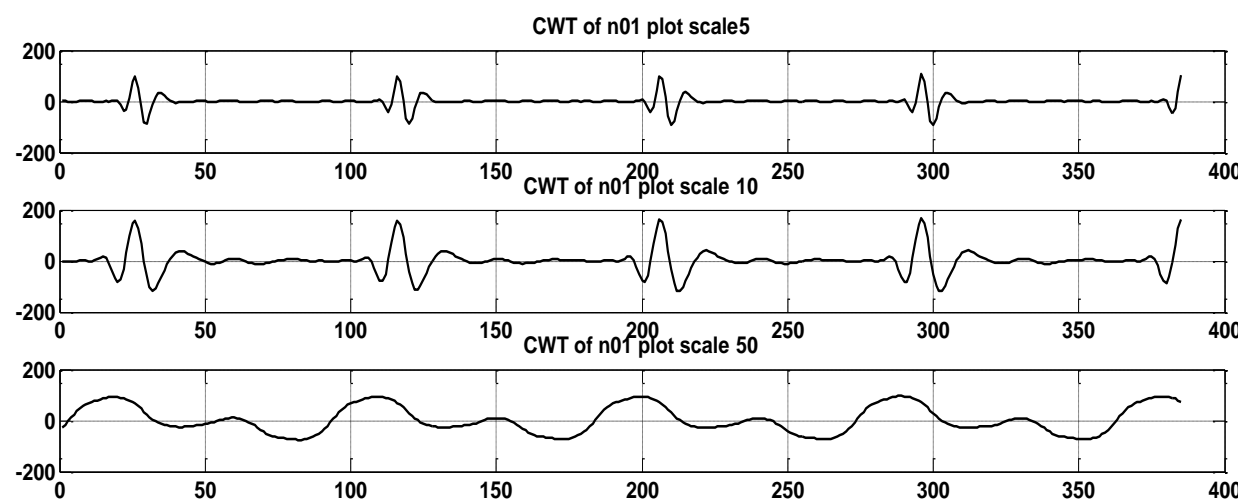

Fig. 3: CWT of the normal record at scales 5, 10, 50 


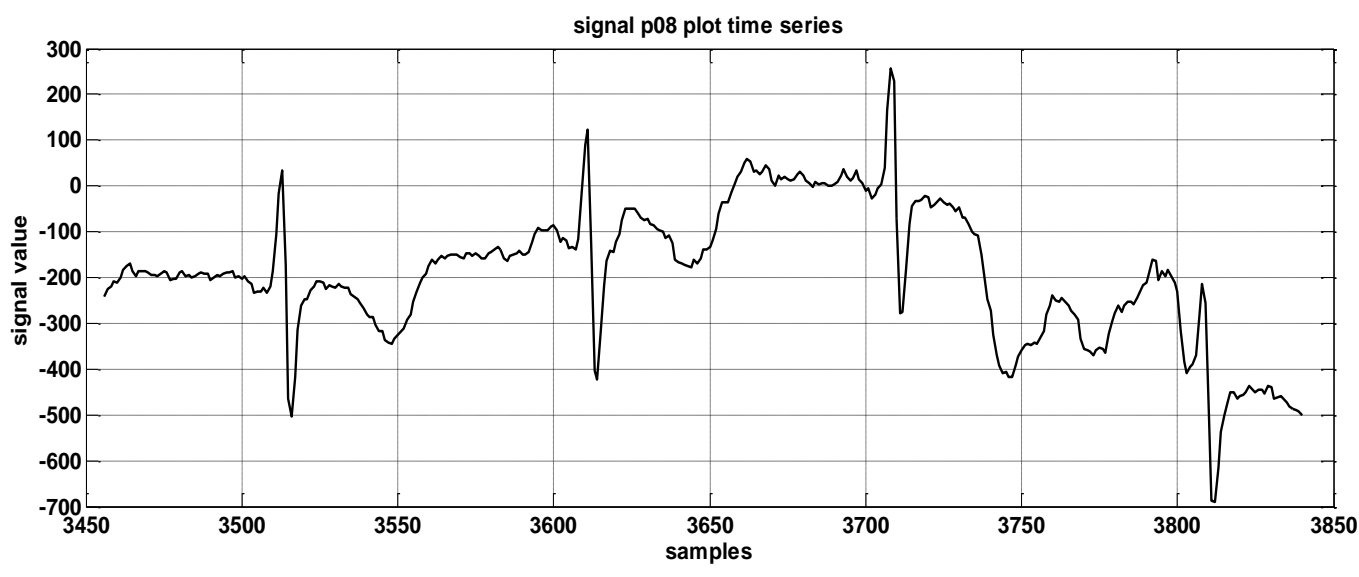

Fig. 4 ECG signal preceding PAF (record p08)
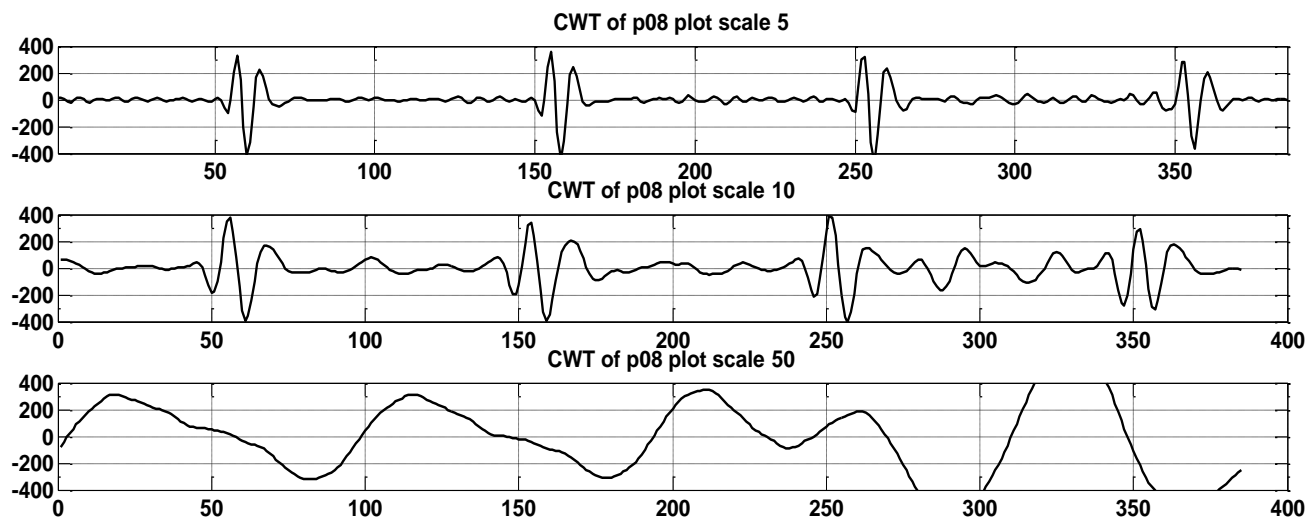

Fig. 5: CWT of the PAF record at scales 5, 10, 50

\subsection{Feature Extraction.}

We utilized two feature sets: FS-1, (1- 3) related to signal statistical and FS-2, (4-11) by mean of continuous wavelet transform as follows:

(1) Sigmean: mean value of the signal during all period (30 $\mathrm{min})$, the histogram of the obtained results is illustrated in Fig. 6. We can note that most of PAF records have a high negative value.

(2) Sigstd: the standard deviation of the recorded signal, the histogram of the obtained results is illustrated in Fig. 7. We can note that most of PAF records have a high positive value.

(3) Sigdiff: difference between the maximum signal and the minimum signal, the histogram of the obtained results is illustrated in Fig. 8. We can note that most of PAF records have a small positive value.

(4) RRno: number of RR interval inside each period. We first detect R peak inside each period, and with the aid of CWT, we can discriminate the extremes values and their locations.

(5) RRdiff: The difference between max. RR interval and min. RR interval inside each period.

(6) RRmax: maximum values of RR interval inside each period.

(7) RRmean: mean values of RR interval inside each period.

(8) RRstd: standard deviation of RR interval inside each period.

(9) RRrms: root mean square values of RR interval inside each period.

(10) Ramp: mean value of $\mathrm{R}$ peak inside each period.

(11) QRSmean: mean value of QRS duration inside each period. 


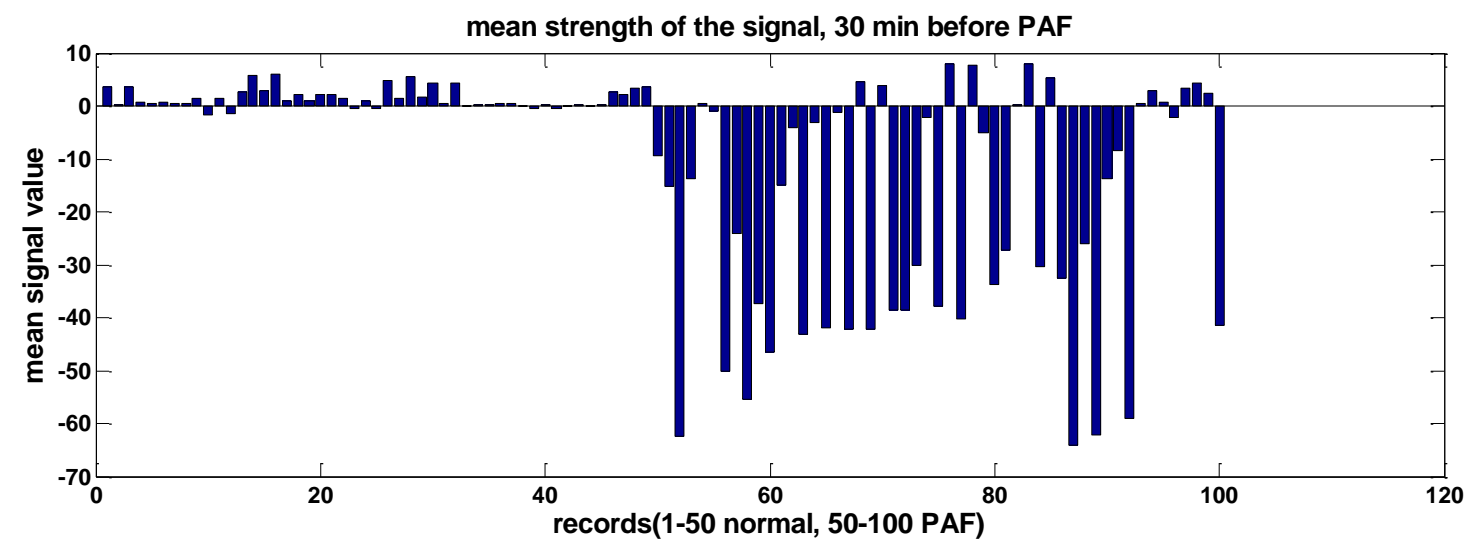

Fig. 6: Histogram of the mean signal for all records.

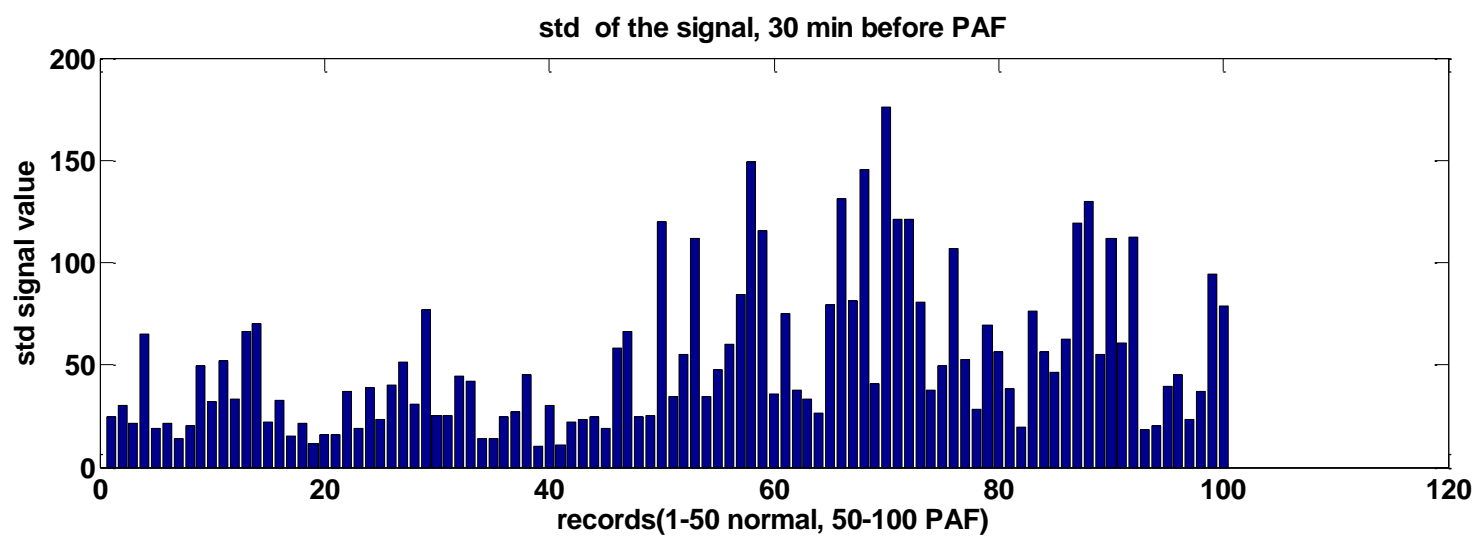

Fig. 7: Histogram of the standard deviation of the signal for all records.

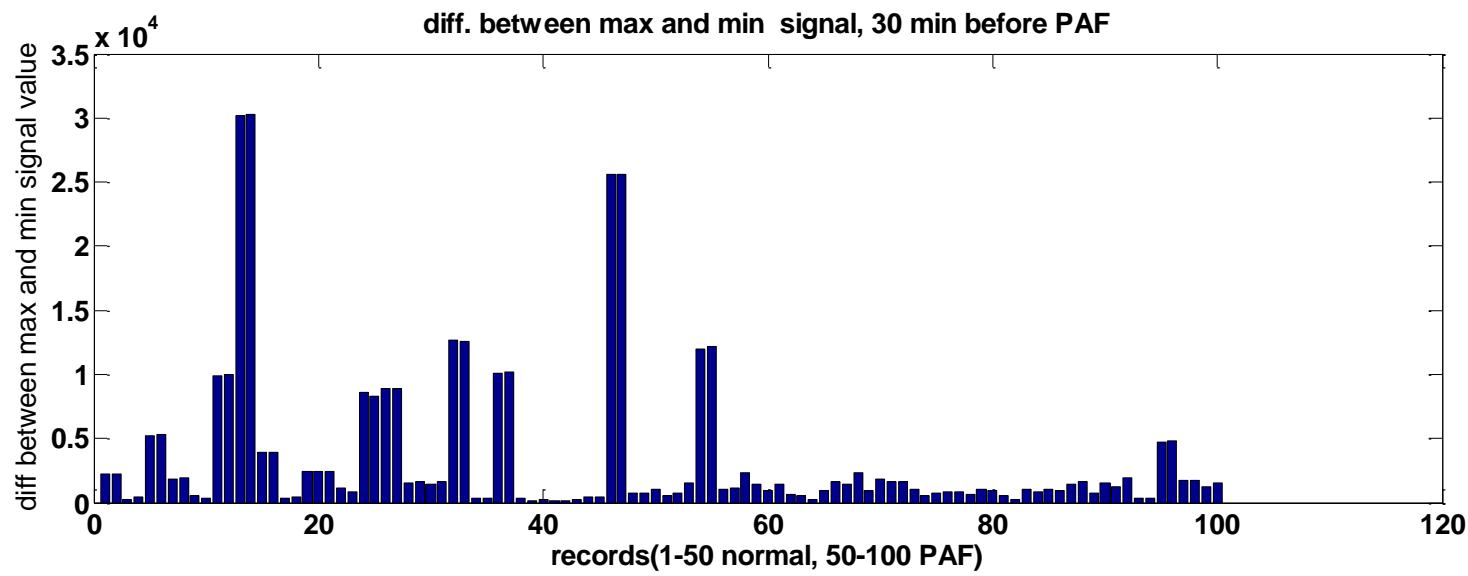

Fig. 8: Histogram of the signal difference between (max. and min.) signal for all records.

\subsection{Neural Network Classifier.}

A supervised ANN is developed to recognize and classify the features of ECG signal for normal and PAF record. Typically, multilayer feed-forward neural network (newff) can be trained as non-linear classifier using the generalized back propagation algorithm (BPA). The BPA is a supervised learning algorithm, in which a sum square error function is defined, and the learning process aims to reduce the overall system error to a minimum. The architecture of the neural network used in this work is the pattern recognition neural network (newpr), (newpr), returns a network exactly as (newff) would, but with an output layer transfer function of 'tansig' and additional plotting functions included in the network's net.plotFen property. The Network include 10, 5 hidden neurons in the second and third layer respectively. The number of input nodes are determined by the finalized data; the number of hidden neurons are determined through trial and error; the number of output nodes are 1 in case of 'newpr' since we have only two classes.

\section{RESULTS AND DISCUSSION}

To evaluate the performance of the proposed method during 30 -min preceding the (PAF), we divide 30 -min period into 6 intervals, 5-min each. Four criterias are used as follows:

Sensitivity $(\%)=\frac{T P}{T P+F N} 100$

Specificity $(\%)=\frac{T N}{T N+F P} 100$ 
PositivePredicitivity $(\%)=\frac{T P}{T P+F P} 100$

Accuracy $=\frac{T P+T N}{T P+T N+F P+F N} 100$

Where:

TP: True Positive, when an object having (PAF) is classified correctly.

TN: True Negative when a normal object is classified correctly. FN: False Negative when an object having (PAF) is classified as normal incorrectly

FP: False Positive when a normal person is classified as having PAF incorrectly

To evaluate the performance of the proposed classifier (NEWPR), we used 60 records ( $30 \mathrm{n}$ and $30 \mathrm{p}$ ) for training and 40 records $(20 \mathrm{n}$ and $20 \mathrm{p})$ for testing. The four previously mentioned measures are calculated in each 5-min interval. To confirm the obtained results, we perform 100 run simulations for the (NEWPR), and then take the mean value for the mentioned measures. The mean values of the criteria's for each 5- min interval are tabulated in Table 1.

The result of (NEWPR) output classifier and performance, in training, validation and testing data set are illustrated in Fig. 9 and Fig. 10 respectively.

We can deduce the following points from analysis of the obtained results:

- We can predict PAF efficiently even in 30 min prior to PAF
- $\quad$ The average percentage of the sensitivity, specificity, positive predictivity and accuracy are higher during 5-min interval preceding the PAF directly, and also during 5-min interval, 20-min, prior PAF

- The efficiency of the CWT to allow accurate extraction of features from non-stationary signal like ECG.

- Robustness of (NEWPR) classifier to handle large feature spaces

- $\quad$ Features like sigmean, sigstd, sigdiff, RRno, RRdiff Ramp and QRSmean enhance the (NEWPR) to distinguish between normal ECG record and PAF ECG record

The comparison between the obtained results and other results, in the same field, in the literature $[8,11,12]$

is shown in Table 2.

\section{CONCLUSION}

ANN-based classifier is introduced in this work. We extract two feature sets, feature set- 1 directly from ECG signal and feature set- 2 with the aid of CWT, to allow accurate extraction of feature from non-stationary signal like ECG, from 100 ECG recorded signals of 'afpdp' database. (NEWPR) is used to classify the patterns inherent in the features extracted, into normal record and PAF record. The results show that we can predict the PAF accurately in 5-min \& 20-min prior the PAF. The obtained results show the efficiency of the proposed method in predicting the onset of PAF. The average percentage of sensitivity, specificity, positive predictivity and accuracy are $91.7 \%, 94.5 \%, 95.8 \%$, and $93.1 \%$ respectively, and these values outperform the obtained results in the literature

Table 1. Mean value of the criteria's

\begin{tabular}{|c|c|c|c|c|}
\hline Period (5min) & Sensitivity (\%) & Specificity (\%) & Positive predicit. (\%) & Accuracy (\%) \\
\hline $30 \mathrm{~min}$ prior to $\mathrm{PAF}$ & 82.0792 & 95.0495 & 95.8621 & 88.5644 \\
\hline 25 min prior to $\mathrm{PAF}$ & 81.7647 & 91.2745 & 93.4164 & 86.5196 \\
\hline 20min prior to $\mathrm{PAF}$ & 90.7843 & 93.4314 & 94.3567 & 92.1078 \\
\hline 15 min prior to $\mathrm{PAF}$ & 83.8235 & 88.2353 & 89.4149 & 86.0294 \\
\hline 10 min prior to $\mathrm{PAF}$ & 85.1961 & 83.3333 & 85.5185 & 84.2647 \\
\hline $5 \mathrm{~min}$ prior to $\mathrm{PAF}$ & 91.7327 & 94.5545 & 95.8037 & 93.1436 \\
\hline
\end{tabular}



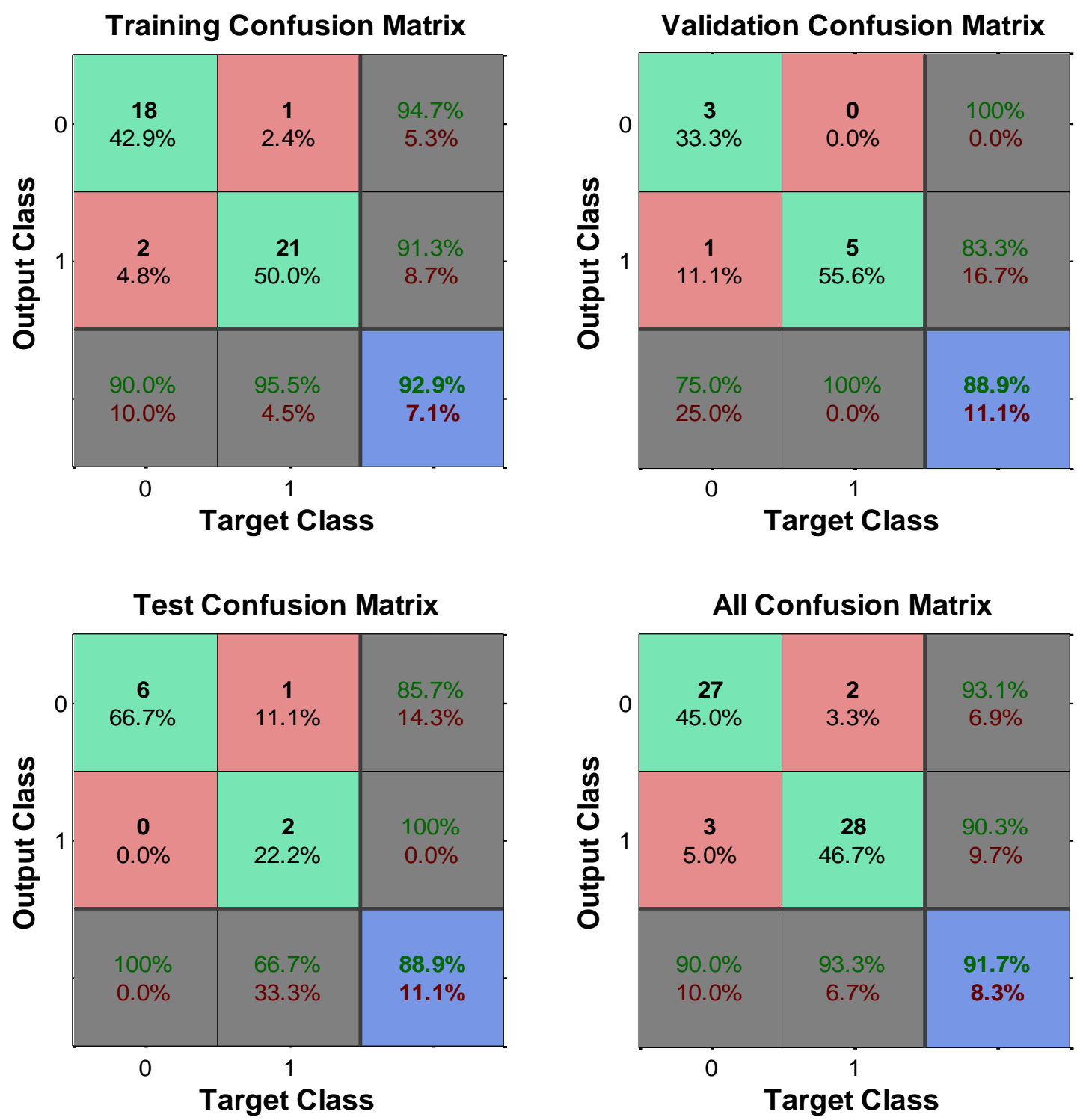

Fig. 9: NEWPR output classification results

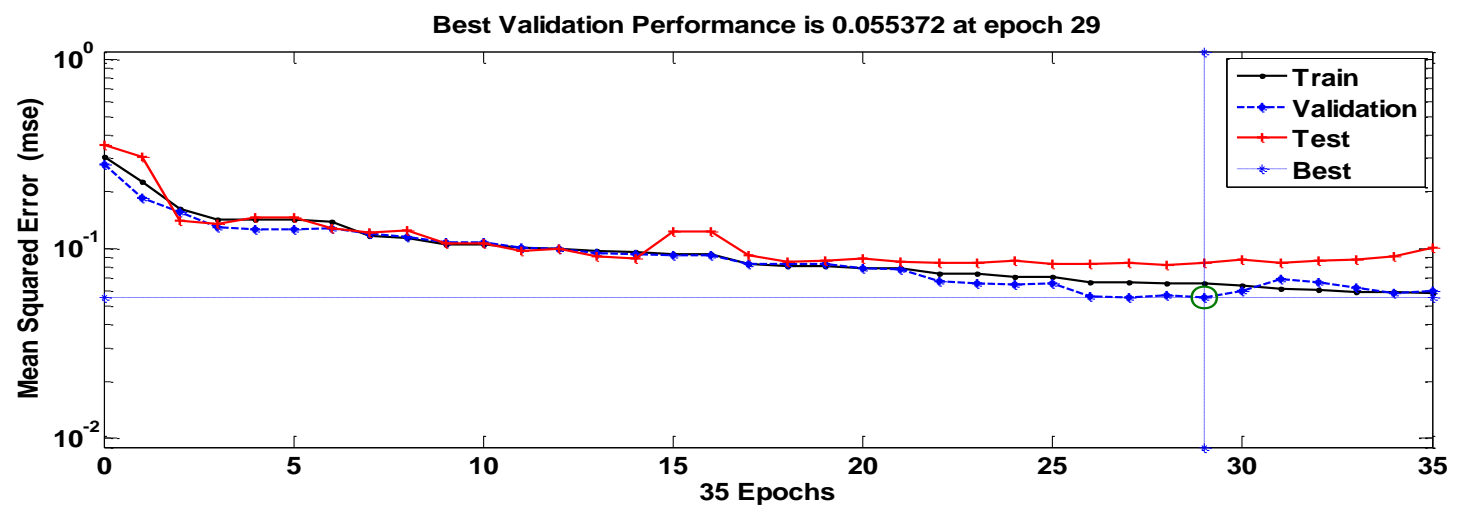

Fig.10: NEWPR training, validation and test performance 
Table 2. Comparative results in the literature

\begin{tabular}{|c|c|c|c|c|}
\hline Method & Literature & Sensitivity (\%) & Specificity (\%) & Accuracy (\%) \\
\hline HRV & Hariton Costin, et al., 2013 [8] & 84.51 & 83.93 & - \\
\cline { 1 - 3 } MV & & 87.32 & 87.5 & - \\
\cline { 1 - 4 } HRV+MV & B.Pourbabaee, et al., 2008 [11] & 89.44 & 89.29 & - \\
\hline $\begin{array}{c}\text { MLP network } \\
\text { results }\end{array}$ & M. Panusittikorn, et al., 2010 [12] & - & - & 87.5 \\
\hline $\begin{array}{c}\text { K-nearest neighbor } \\
\text { algorithm }\end{array}$ & Ashraf, 2013 & 91.0 & 65.0 & - \\
\hline \begin{tabular}{c} 
Proposed method \\
\cline { 3 - 4 }
\end{tabular} & & 94.5545 & 93.1436 \\
\hline
\end{tabular}

\section{REFERENCES}

[1] Bollmann A., Husser D., Mainardi L. et al., Analysis of Surface Electrocardiograms in Atrial Fibrillation: Techniques, Research, and Clinical Applications. Europace, 8, 911-926, 2006.

[2] Chiarugi F., New Developments in the Automatic Analysis of the Surface ECG: the Case of Atrial Fibrillation. Hellenic J. of Cardiology, 49, 207-221, 2008.

[3] Sörnmo L., Stridh M., Husser D., Bollmann A., Bertil Ollsen S., Analysis of Atrial Fibrillation: from Electrocardiogram Signal Processing to Clinical Management. Phil. Trans. of the Royal Society, A, 367, 235-253, 2009.

[4] S.Karpagachelvi, et al., ECG Feature Techniques- A Survey Approach. International Journal of Computer Science and Information Security, 8, 1, 76-80, 2010

[5] Moody G.B., Goldberger A.L., McClennen S., Swiryn S.P., Predicting the Onset of Paroxysmal Atrial Fibrillation: the Computers in Cardiology Challenge 2001. Computers in Cardiology, 28, 113-116, 2001.

[6] De Chazal P., Heneghan C., Automated Assessment of Atrial Fibrillation. Computers in Cardiology, 28, 117-120, 2001.

[7] Maier C., Bauch M., Dickhaus H., Screening and Prediction of Paroxysmal Atrial Fibrillation by Analysis of Heart Rate Parameters. Computers in Cardiology, 28, 129-132, 2001.

[8] Hariton Costin, et al. A New Method for Paroxysmal Atrial Fibrillation Automatic Detection. Buletinul Institutului Politehnic din Iaşi Tome LIX (LXIII) Fasc. 1, 2013
[9] S. Issac Niwas, et al., Artificial Neural Network Based Automatic Cardiac Abnormalities Classification.

[10] D. Shanthi, et al., Designing an Artificial Network Model for the Prediction of Thrombo-embolic Stroke. International Journals of Biometric abd Bioinformatics (IJBB), 3, 1, 10$18,2010$.

[11] B. Pourbabaee, C.Lucas, Automatic Detection and Prediction of Paroxysmal Atrial Fibrillation Based on Analyzing ECG Signal Feature Classification Methods. Proceedings of the 2008 IEEE, CIBEC'08, 2008.

[12] M. Panusittikorn, et al., Prediction of Paroxysmal Atrial Fibrillation Occurrence with Wavelet-based Markers. IEEE International Conference, 342-345, 2010.

[13] A. K. M. Fazlul Haque, et al., Detection of Small Variations of ECG Features Using Wavelet. ARPN Journal of Engineering and Applied Sciences, 4, 6, 2009

[14] Digvijay, et al., Wavelet Aided SVM Analysis of ECG Signals for Cardiac Abnormality Detection. IEEE Indicon conference, Chennai, India, 9-13, 2005.

[15] Erik Zellmer, et al., Highly Accurate ECG Beat Classification based on Continuous Wavelet Transformation and Multiple Support Vector Machine Classifiers. IEEE International Conference, 1-5, 2009.

[16] Proceedings of the Sixth International Conference on Computational Intelligence and Multimedia Applications (ICCIMA'05), IEEE Computer Society, 2005. 